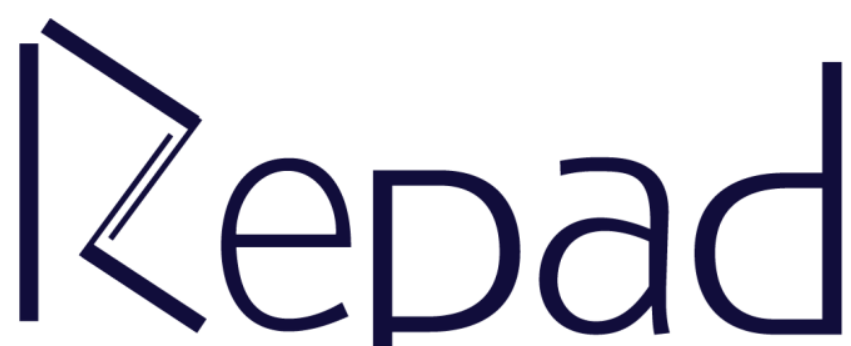

v. 4, n. 2, Maio-Agosto/2020

Revista Estudos e

Pesquisas em Administração 


\section{A ELASTICIDADE DE TRANSMISSÃO DE PREÇOS ENTRE OS ELOS DE MERCADO: Produtor, atacado e varejo na comercialização da carne bovina no período de 1997 a 2013}

Anderson Nunes de Carvalho Vieira andersonvieira.nunes@hotmail.com https://orcid.org/0000-0002-4200-9420 Faculdade de Tecnologia do SENAI-MT

Cuiabá-Mato Grosso, Brasil

Andrey Sartori https://orcid.org/0000-0001-6882-3378 Faculdade de Tecnologia do SENAI-MT Cuiabá- Mato Grosso, Brasil

Fabrício César de Moraes https://orcid.org/0000-0002-0183-1178 Instituto Federal de Educação, Ciência e Tecnologia de Mato Grosso

Cuiabá- Mato Grosso, Brasil

Rosicley Nicolao de Siqueira http://orcid.org/0000-0001-7944-1084 Faculdade de Tecnologia do SENAI-MT Cuiabá- Mato Grosso, Brasil

\section{Resumo}

O agronegócio brasileiro contribui essencialmente para o desenvolvimento econômico do país. Inserida nesse complexo está a cadeia de carne bovina com um percentual significativo para as exportações brasileiras impulsionando a economia através de sua participação no consumo interno de alimentos. Neste trabalho, analisa-se a elasticidade de transmissão de preços entre os elos de mercado, na cadeia da carne bovina. O modelo proposto foi implementado utilizando-se teste de raiz unitário de Dickey-Fuller Aumentado (ADF), teste de cointegração de Johansen e método de Vetor Auto Regressivo (VAR) e Vetor de Correção de Erros (VEC), Decomposição da Variância e finaliza-se com a função de impulso resposta com o choque de $1 \%$ nos elos dentro das cadeias estudadas. Os dados analisados são referentes aos preços da carne de boi praticados no atacado, no varejo e ao produtor, entre julho de 1997 e dezembro de 2013. Observa-se, de acordo com os resultados encontrados, que o atacado é o principal responsável pela transmissão de preços entre os demais elos, ou seja, choques de preço ocorridos no atacado são transmitidos de forma significativa para o produtor e o varejo.

Palavras-chave: Agronegócio, Elasticidade, Transmissão de Preços. 
THE ELASTICITY OF PRICE TRANSMISSION BETWEEN MARKET LINKS: Producer, wholesale and retail in the marketing of beef in the period from 1997 to 2013

\begin{abstract}
Brazilian agribusiness contributes essentially to the economic development of the country. Included in this complex is the beef chain with a significant percentage for Brazilian exports boosting the economy through its share of domestic food consumption. In this work, the elasticity of price transmission between the market links in the beef chain is analyzed. The proposed model was implemented using the DickeyFuller Increased unit root (ADF) test, Johansen cointegration test and (VAR), Variance Decomposition Vector (VEC), Variance Decomposition and finalization with the impulse response function with the $1 \%$ shock at the links within the studied chains. The data analyzed refer to the prices of beef practiced in the wholesale, retail and producer between July 1997 and December 2013. It is observed, according to the results found, that the wholesale is the main responsible for the transmission between the other links, that is, wholesale price shocks are transmitted significantly to the producer and the retailer.
\end{abstract}

Keywords: Agribusiness, Elasticity, Price Transmission.

Submetido: $30 / 03 / 2020$

Aceito: $15 / 05 / 2020$

Publicado: 31/05/2020

\title{
INTRODUÇÃO
}

O cenário atual aponta que o Brasil se destaca consideravelmente na produção e comercialização de produtos agropecuários, sendo o agronegócio uma das mais importantes fontes geradoras de riquezas no país. Segundo Barros (2006), houve um grande incremento qualitativo e quantitativo dos produtos brasileiros na última década, o que ocorreu devido ao crescimento sistemático na qualidade e controle da produção e do processamento produtivo. Além disso, o Brasil apresenta condições que favorecem esse desenvolvimento, a exemplo de chuvas regulares, clima diversificado, energia solar abundante, aproximadamente $13 \%$ de toda a água doce disponível no planeta, entre outros (EMBRAPA, 2009).

Esses fatores fazem do país um lugar de vocação natural para a produção de todos os negócios relacionados às suas cadeias produtivas. De acordo com informações da Empresa Brasileira de Pesquisa Agropecuária (EMBRAPA, 2009), o Brasil construiu um sistema produtivo altamente eficiente $\mathrm{e}$ competitivo no que se refere ao agronegócio. Conforme Barros (2015), em dezembro de 2014 os exportadores do agronegócio brasileiro obtiveram receita na ordem de US\$ 6,8 bilhões, alta de $6 \% \mathrm{em}$ relação a dezembro de 2013, segundo estatísticas do Centro de Estudos Avançados em Economia Aplicada - CEPEA-ESALQ/USP (2015).

$\mathrm{Na}$ cadeia produtiva do agronegócio está inserida a produção de carne bovina, que atingiu um nível de exportação de $3 \%$ em 2009, totalizando um faturamento de 6 bilhões de reais. Tal produto representa $6 \%$ do Produto Interno Bruto (PIB), ao mesmo tempo que equivale à $30 \%$ do PIB do agronegócio brasileiro, movimentando mais de 
400 bilhões de reais. Vale ressaltar que estes valores aumentaram em quase $45 \%$ nos últimos 5 anos (EMBRAPA, 2009).

Diante do exposto o presente trabalho tem como objetivo principal analisar a elasticidade de transmissão de preços entre os elos de comercialização no mercado brasileiro da carne bovina baseando-se nas séries temporais do estado de São Paulo. Desta forma, a pergunta a ser respondida no presente estudo é: Qual dos três níveis de mercado (atacado, produtor ou varejo) tem a maior influência sobre os preços nos demais níveis?

O processo de comercialização, envolvendo os três níveis de mercado, produtor, atacado e varejo, desenvolve-se a partir do momento que os bens são transferidos dos produtores aos consumidores, cujos resultados dessas trocas são as transações responsáveis pelo desenvolvimento e funcionamento de um sistema econômico. Além disso, a hipótese a ser investigada será o atacado na carne bovina, por trabalhar com maior escala no comércio do produto, que poderá assumir o papel de líder na transmissão de choques de preços para os demais elos.

\section{FUNDAMENTAÇÃO TEÓRICA}

\subsection{Elasticidades de Preço e o Processo de Comercialização}

A elasticidade refere-se ao grau de sensibilidade que uma variável tem em virtude de alterações em outra variável. Assim, de acordo com Mankiw (2010), na lei de demanda, quando há uma queda no preço de um bem o que se espera é que exista um aumento na quantidade demandada. Sendo assim, consequentemente, a elasticidadepreço da demanda é utilizada para medir o quanto a quantidade demandada reage dada uma variação no preço, ou seja, o quanto e como os consumidores estão dispostos a deixar de comprar determinado bem à medida que seu preço aumenta. Se a demanda de determinado produto for elástica, significa que os consumidores desse produto possuem grande reação às variações do preço, ou seja, o aumento do preço reduz proporcionalmente mais a quantidade demandada. Entretanto, quanto mais produtos substitutos existirem, maior será a elasticidade. A demanda infinitamente elástica é uma situação de mercado em que os consumidores irão adquirir a quantidade que puderem de certo bem, a determinado preço; e a qualquer preço mais alto do que esse, a demanda cai a zero, outrossim, a qualquer preço mais baixo, a demanda aumenta ilimitadamente (PYNDICK, RUBINFELD, 2010).

A comercialização envolve uma série de atividades, em um processo de produção, onde os bens e serviços são transferidos dos produtores aos consumidores. Essas atividades resultam na transformação dos bens, mediante utilização de recursos produtivos - capital e trabalho - que atuam sobre a matéria-prima agrícola. De acordo com Piza e Welsh (1968, p. 78), a comercialização compreende "o conjunto de atividades realizadas por instituições que se acham empenhadas na transferência de bens e serviços desde o ponto de produção inicial até que eles atinjam o consumidor final".

Conforme Zylberstajn (2000), comercialização seria a troca de bens e serviços entre agentes econômicos. O fruto dessas trocas são as transações que podem ser consideradas a unidade fundamental da análise do funcionamento do sistema econômico. Todo esse processo representado na cadeia produtiva é explicado pela microeconomia por meio de mercado consiste em um grupo de compradores e 
vendedores, que, por intermédio e suas interações efetivas ou potenciais, determinam o preço de um produto ou um conjunto de produtos (PINDYCK, 2010).

Segundo Mendes e Padilha Jr. (2007), entre a produção e o consumo há uma série de funções desempenhadas pelos agentes envolvidos e o processo de comercialização pode ser dividido em três fases distintas: concentração, equilibrio e dispersão. O primeiro passo é a procura e a reunião dos produtos produzidos pelo grande número de produtores, a chamada concentração de mercado. O Segundo passo seria a temos da taxa de equilibrio, onde se sintetizam as atividades que regulam o fluxo de produção (sazonalidade) em função da taxa consumida que é variável no decorrer do tempo. Já o último passo seria a finalização do processo de dispersão representado pelo agrupamento dos grandes lotes de produtos da concentração que é destinado ao consumo, no lugar, no tempo e nas formas desejadas pelos consumidores finais.

Os diferentes níveis de mercado são os responsáveis pelo processo de comercialização dos produtos. Ele subdivide-se em três grandes grupos: produtor, atacadista e varejista (BARROS, 2007). A relação entre esses três níveis ocorre da seguinte forma: os produtores oferecem sua produção aos intermediários. As transações volumosas têm lugar no mercado atacadista, além disso, nesse nível ocorrem as trocas entre intermediários, os atacadistas e varejistas e por outro lado nessa etapa ocorre uma pequena participação de produtores e consumidores. Os varejistas constituem o último elo da cadeia, pois colocam a mercadoria a disposição dos consumidores.

\subsection{Transmissões de Preços}

O estudo da transmissão de preços foi realizado primeiramente por Gardner (1975) em seu modelo analítico para um mercado em concorrência perfeita, que se equilibra a cada mudança de demanda e oferta. O autor desenvolveu um modelo estatístico comparativo para determinar a transmissão de preços entre mercados. Entende-se a transmissão de preços entre dois mercados como uma situação em que qualquer alteração de preço em um mercado é instantaneamente transmitida a outro mercado.

Após alguns anos, o modelo é reproduzido por Barros (1990), acompanhado de análise gráfica aplicável para situações em que se podem admitir funções de coeficientes fixos. De acordo com esse modelo, nos níveis de varejo, de atacado ou de produtor, as variações podem ocorrer, demonstrando que não há um único nível que sistematicamente lidere essas variações. É possível notar que o processo de transmissão de preços está relacionado com a identificação do agente da cadeia relacionando os três agentes acima citados.

Segundo Barros (1990), a liderança está associada à sensibilidade do setor às variações da oferta e demanda, ao custo para alterar os preços, à quantidade de transações com produtos (especialização) e ao risco de prejuízo nas transações. Sob essas condições, a elasticidade de transmissão de preços, na medida dos preços do produtor para o varejo, sempre será inferior ou igual à unidade, ou seja, as variações de preço ao produtor serão sempre maiores ou iguais as variações ao nível de varejo. Por outro lado, Barros (1990) considera que dentre os três níveis de mercado o setor atacadista pode trabalhar com volumes maiores de produtos, podendo assumir a liderança nas variações de preços devido ao maior acesso a informação e ao baixo custo de mudanças de preços. Ele enfatiza que o ajustamento de preço ocorre instantaneamente pelo excesso de demanda. 


\subsection{Cenário Brasileiro da Produção de Carnes Bovinas}

A introdução do gado no Brasil caracteriza-se pelo sistema extensivo de produção, desenvolvendo-se através da expansão das fronteiras agrícolas, com a incorporação de novas terras. Outro fator que impulsionou o crescimento da produção de carnes bovinas foi a incorporação de novas tecnologias, no século $\mathrm{XX}$, visando $\mathrm{o}$ aumento da produtividade, crescendo, assim, sistemas intensivos de produção em determinadas regiões conhecidos como confinamentos e semiconfinamentos. Neste período ocorre também o incentivo governamental com o intuito de estimular novas tecnologias para a importação de raças indianas e europeias. A introdução de sal na alimentação dos bovinos trouxe um controle mais efetivo sobre a febre aftosa, que vinha prejudicando esse mercado. Cabe destacar também que o sucesso do agronegócio é devido, em partes, a uma estratégia desenhada nesse período que apontou para a resolução de vários problemas estruturais que entravavam o desempenho da agricultura e pecuária.

Após esse processo de adaptação do gado, iniciaram-se várias pesquisas para melhorar esse sistema produtivo. A EMBRAPA, com a ajuda de incentivos financeiros, desenvolveu projetos para levar o gado zebuíno ${ }^{1}$ e a braquiária ${ }^{2}$ para a região Norte e Centro-Oeste. Atualmente o elevado crescimento de produção de carne bovina no Brasil posicionou o país diante do mercado internacional. As exportações brasileiras de carne bovina seguem em grande escala. De acordo com os dados da Associação Brasileira das Indústrias Exportadoras de Carnes Bovinas (ABIEC, 2018), de janeiro a março desse ano, os embarques somaram 405.668 toneladas, crescimento $2,6 \%$ em relação às 395.536 toneladas no mesmo período do ano passado. Trata-se do melhor início de ano dos últimos 12 anos. Em receita, as vendas do período somaram US\$ 1,51 bilhão, redução de 5,6\% ante a receita de US\$ 1,60 bilhão no primeiro trimestre de 2018.

$\mathrm{O}$ acentuado aumento das exportações depende de vários fatores, e, entre eles, as pastagens brasileiras que favorecem a criação e atendem a produção da grande quantidade demandada do alimento, provocando melhoras do manejo alimentar do rebanho. De acordo com dados da Pesquisa da Pecuária Municipal (PPM) realizada em 2014 pelo IBGE, o Brasil possui aproximadamente 213,5 milhões de cabeça de gado, sendo que $14 \%$ se concentra no estado de Mato Grosso. Assim, segundo a pesquisa, Mato Grosso é o maior produtor de bovinos do país desde 2004. Para Pereira (2012), esse sucesso já era esperado no estado devido as fatores econômicos e ambientais que favorece esse nicho de mercado do agronegócio mato-grossense. Pereira (2012) também ressalta $\mathrm{o}$ sucesso das políticas macroeconômicas dos últimos governos, $\mathrm{o}$ impulsionamento da Lei Kandir e as parcerias comerciais com a China que contribuíram substancialmente com o sucesso da pecuária mato-grossense.

\section{PROCEDIMENTOS ECONOMÉTRICOS ADOTADOS}

O presente trabalho constitui-se a partir do levantamento de dados secundários, referentes aos preços mensais das carnes nos três níveis de mercado, entre os anos de 1997 a 2013. Os dados foram coletados no site do Instituto de Economia Agrícola do Estado de São Paulo - IEA/SP. Os preços foram deflacionados pelo Índice Geral de

\footnotetext{
${ }^{1}$ Zebuíno ou Zebu é uma subespécie de bovino descendente do Bos Taurus Indicus. É umdos tipos de bovinos que mais se adaptaram ao cerrado brasileiro.

${ }^{2}$ Braquiária é uma espécie de planta pertencente à família Poaceae, muito utilizada para alimentação de ruminantes no Centro-Oeste brasileiro.
} 
Preços Disponibilidade Interna - IGP-DI, da fundação Getúlio Vargas - FGV, com base em dezembro de 2013. Para que seja eliminado o efeito da inflação sobre os valores nominais da série de dados, precisa-se deflaciona-la, para assim consequentemente obter o real poder aquisitivo em relação ao ano base. A cadeia produtiva estudada refere-se à média no Brasil, baseando-se no estado de São Paulo, pois representa o maior polo de comercialização do país. Para a realização dos cálculos econométricos foram utilizados os programas $R$ e WINRATS.

\subsection{Testes de Raiz Unitária}

O primeiro passo antes da aplicação do método proposto é verificar se as séries utilizadas são estacionárias. De acordo com Bueno (2011), uma série é estacionária quando possuir média e variância constante ao longo do tempo e covariância dependente apenas das defasagens entre períodos de tempo (e não do período de tempo em que a covariância é calculada). A ordem de integração da variável, por sua vez, diz respeito ao número de vezes que uma série deve ser diferenciada $\left(X_{t-i}-X_{t}\right)$, para que se torne estacionária. Conforme Gujarati (2006), as propriedades de uma série estacionária, algebricamente, são as seguintes:

$$
\begin{aligned}
& E\left(Y_{t}\right)=\mu \\
& \operatorname{var}\left(Y_{t}\right)=E\left(Y_{t}-\mu\right)^{2}=\sigma^{2} \\
& \gamma_{k}=E\left[\left(Y_{t}-\mu\right)\left(Y_{t+k}-\mu\right)\right]
\end{aligned}
$$

De acordo com Santana (2003), uma representação de um processo não estacionário corresponde ao modelo de raiz unitária, ou seja, quando o processo gerador dos dados possui uma raiz unitária (ou tendência estocástica). Um teste muito utilizado na prática para verificação de raiz unitária foi proposto pelos pesquisadores Dickey e Fuller (1981), conforme resumido a seguir.

Suponha um modelo do tipo AR (1):

$$
y_{t}=y_{t-1}+\varepsilon_{t}
$$

A condição para que esta série seja estacionária é: $\left|a_{1}\right|<1$

Se $a_{1}=1$ diz-se que esta série possui uma raiz unitária (ou tendência estocástica) e, portanto, não será estacionária.

A tendência inicial é estimar esse modelo e usar um teste de hipóteses do tipo $t$ sobre $\mathrm{a}_{1}$, tendo como hipótese nula $H_{0:}$ al $=1$.

Porém, em geral, os pacotes econométricos reportam os testes nos coeficientes contra a nula de serem iguais a zero. Então, o teste poderia ser alterado, subtraindo $y_{t-1}$ de ambos os lados para deixar o parâmetro da hipótese nula igualado à zero.

Subtraindo $y_{t-1}$ de ambos os lados:

$$
\begin{aligned}
& y_{t}-y_{t-1}=a_{1} y_{t-1}-y_{t-1}+\varepsilon_{y t} \\
& \Delta y_{t}=\left(a_{1}-1\right) y_{t-1}+\varepsilon_{y t} \\
& y_{t}=\gamma y_{t-1}+\varepsilon_{y t}
\end{aligned}
$$


Onde: $\left(a_{1}-1\right)=\gamma$

O teste de Dickey e Fuller vai verificar se $\gamma=0$. Se $\gamma$ for igual à zero é por que a série não é estacionária (possui uma raiz unitária). Há três modelos de testes Dickey e Fuller, que são: Com constante e com tendência determinística em que o valor crítico é dado pela estatística $\tau_{\tau}$ a:

$$
\Delta y_{t}=a_{0}+a_{2} t+\gamma_{1} y_{t-1}+\varepsilon_{y t}
$$

Com constante, sem tendência determinística em que o valor crítico é dado pela estatística $\tau_{\mu}$ :

$$
\Delta y_{t}=a_{0}+\gamma_{1} y_{t-1}+\varepsilon_{y t}
$$

Sem constante e sem tendência determinística sendo que o valor crítico é dado pela estatística $\tau$ :

$$
\Delta y_{t}=\gamma_{1} y_{t-1}+\varepsilon_{y t}
$$

Sem constante e sem tendência determinística, para séries em primeira diferença:

$$
\Delta^{2} y_{t}=\beta 1 \Delta y_{t-1}+\varepsilon_{t}
$$

Ao confirmar que a série possui pelo menos uma raiz unitária, é necessário diferenciá-la uma vez e repetir o teste para verificar se ela se torna estacionária em primeira diferença, ou seja, para verificar se a variável é integrada de ordem 1. Para testar a variável em primeira diferença, utiliza-se somente o formato mais simples, sem tendência determinística e sem constante, pois a diferenciação elimina os dois componentes.

\subsection{Testes de Cointegração - Modelo Proposto por Johansen (1995)}

Uma vez confirmado que as variáveis em questão são estacionárias em I (1), é necessário verificar se as mesmas são cointegradas, para que se possa utilizá-las conjuntamente em um modelo de Auto-Regressões Vetoriais - VAR. O teste recomendado para verificar a cointegração quando se trabalha com mais do que duas variáveis foi proposto por Johansen (1995).

As estatísticas propostas por Johansen (1995) para determinar as relações de cointegração são calculadas pelos autovalores obtidos, por meio da razão de verossimilhança, cujos testes são a estatística traço e de Máximo, de acordo com SANTANA (2003):

$$
\begin{aligned}
& Q_{r}=-T \Sigma^{k}{ }_{i=1+r} \log \left(1-\lambda_{i}\right) \text { para } r=0,1, \ldots k-1 \\
& Q_{m}=-T * \log \left(1-\lambda_{i+1}\right)=Q r-Q r+1
\end{aligned}
$$


(...) $\mathrm{Q}_{\mathrm{r}}$ e $\mathrm{Q}_{\mathrm{m}}$ são as estatísticas traço e de máximo autovalor, respectivamente; $r$ é o rank de cointegração; $k$ é o número de variáveis endógenas; $\lambda$ é o iésimo maior autovalor (ou raiz característica) e T é o número de observações (Santana, 2003, p. 71).

Neste teste, as relações ocorrem sequencialmente, variando de 0 a $k-1$. O teste é realizado até que se rejeite a hipótese nula. A estatística deste teste é conhecida como estatística traço $\left(Q_{r}\right)$. A hipótese nula prevê que não existe relação de cointegração entre as variáveis, portanto, as variáveis serão cointegradas em uma determinada ordem somente se a hipótese nula for rejeitada. Sendo as hipóteses de acordo com Santana (2003):

$$
\begin{aligned}
& H_{0: r}=0 \\
& H_{a: r}>0
\end{aligned}
$$

e cuja indicação é de que "a análise de acordo com a rejeição da hipótese nula implica que duas séries, $Y_{t}$ e $X_{t}$, são cointegradas, o que é determinante na dinâmica das relações no longo prazo entre as séries apresentadas" (SANTANA, 2003, p. 91).

Com a finalidade de determinar o número de defasagens do modelo VAR, no presente trabalho, foram adotados três critérios: o primeiro, a decisão pelo número de defasagens que minimizou os Critérios de Informação de Akaike (AIC); o segundo foi o de Schwarz (SC) e, por último, o de Hannan-Quinn (HQ).

\subsection{Vetor Auto Regressivo (VAR) e Vetor de Correção de Erros (VEC)}

Posteriormente, ao detectar a relação de cointegração entre as variáveis, direciona-se para a próxima etapa, que consiste na inclusão do vetor de correção de erros. Proposta inicialmente por Cristopher Sims em 1980, Vetor Auto Regressivo (VAR), de acordo com Gujarati (2006), apresenta o termo vetorial em função de tratar de duas ou mais variáveis, e o termo auto regressão devido ao valor defasado da variável dependente.

Complementarmente, como descreve Bacchi (1994), a capacidade deste modelo em estimar modelos que envolvem inter-relações complexas entre as variáveis é muito eficaz, sendo capaz de fornecer a geração de parâmetros que podem ser utilizados em análises prospectivas.

O VAR consiste em um sistema de equações, em que cada variável que compõe o sistema é função dos valores das demais variáveis no presente e valores das demais variáveis defasadas no tempo, mais o termo de erro. As equações de um modelo VAR podem conter também, segundo Enders (1995), tendências determinísticas e variáveis exógenas.

Além de informar sobre as relações de equilibrio em longo prazo entre duas ou mais séries temporais, o conceito de cointegração tem implicação direta sobre as representações dinâmicas por meio dos vetores auto regressivos (SANTANA, 2003).

O sistema VAR contém um conjunto de variáveis, que no modelo assumem o papel de uma função linear dela mesma defasada e também, em função da outra (s) variável do modelo defasada. As equações do modelo VAR estão expressas de acordo com as equações 14 e 15, conforme Gujarati (2006).

$$
P_{t}=\delta+\sum_{j=1}^{n} \beta_{j} P_{t-j}+\sum_{j=1}^{n} \gamma_{j} F_{t-j}+u_{1 t}
$$




$$
P_{t}=\omega+\sum^{n}{ }_{j=1} \theta_{j} P_{t-j}+\sum^{n}{ }_{j=1} \lambda_{j} F_{t-j}+u_{2 t}
$$

Para o VEC, há a inserção de um vetor de correção de erro de forma que o vetor de correção de erro é incorporado em um VAR com variáveis em diferença da seguinte forma:

$$
\begin{array}{r}
\Delta y_{t}=a_{1}+a_{0 y y t}-1 \beta y_{t-1}+i=1 \alpha 11(i) \Delta y_{t-i}+i=1 \alpha 121 \Delta z_{t-i}+\varepsilon_{y t} \Delta z_{t}=\alpha_{1}+ \\
\alpha_{z}\left[y_{t-1}-\beta y_{t-1}\right]+\sum_{i=1} \alpha 21 \text { (i) } \Delta y_{t-i}+\sum_{i=1 \alpha 22(1) \Delta z_{t-i}+\varepsilon_{z t}}
\end{array}
$$

Em que $\left[y_{t-1}-\beta y_{t-1}\right]$ é a correção de erro incorporado no modelo.

\subsection{Análise da Decomposição da Variância e Impulso resposta}

A partir do VAR, as análises são realizadas em grande parte via observação da decomposição da variância e do impulso resposta. A decomposição da variância permite avaliar o poder explanatório de cada variável do VAR/VEC sobre as demais, identificando a proporção de movimentos de uma série que é devida a choques nela mesma, bem como a proporção que é devida aos choques que ocorrem nas outras variáveis inseridas no sistema. Conforme Margarido (2000), "a análise da decomposição da variância mostra a evolução do comportamento dinâmico apresentado pelas variáveis do sistema econômico, ao longo do tempo, isto é, permite separar a variância dos erros de previsão para cada variável em componentes que podem ser atribuídos por ela e pelas demais variáveis endógenas isoladamente, apresentando em termos percentuais qual o efeito que o choque não antecipado sobre determinada variável tem sobre ela mesma e sobre as demais variáveis pertencentes ao sistema".

De acordo com Pindyck e Rubinlfed (2010), a metodologia VAR oferece um meio de deixar os próprios dados determinarem a estrutura dinâmica de um modelo. A função impulso-resposta avalia o impacto que um choque em cada variável causa sobre si mesma, bem como nas demais variáveis do modelo, nos períodos seguintes ao que ocorreu o choque.

\section{RESULTADOS E DISCUSSÕES}

\subsection{Testes de raiz unitária}

Conforme já mencionado, para verificar a estacionariedade das séries utilizadas no presente estudo foi utilizado o teste de raiz unitária, proposto por Dickey e Fuller (1981).

Tabela 1. Resultado dos testes de Raiz Unitária para a cadeia da carne bovina

\begin{tabular}{lcccc}
\hline & & Em nível & & Em diferença \\
\hline Variável & $\tau_{\tau}$ & $\tau_{\mu}$ & $\tau$ & $\tau$ \\
\hline Boiatac & $-1,80$ & $-1,82$ & 0,24 & $-3,32$ \\
Boivar & $-1,28$ & $-0,87$ & 0,48 & $-3,15$ \\
Boiprod & $-2,67$ & $-2,66$ & 0,22 & $-3,52$ \\
\hline
\end{tabular}

Fonte. Resultados da pesquisa. Nota. Valores críticos a 1\% de significância, em Fuller (1976):

$$
\tau_{\tau}=-3,99 / \tau_{\mu}=-3,46 / \tau=-2,58
$$

Os resultados da Tabela 1 mostram que todas as séries consideradas na análise são integradas de ordem 1, denominadas I (1), pois não são estacionárias em nível, mas se tornam estacionárias em primeira diferença. 


\subsection{Teste de cointegração de Johansen}

$\mathrm{O}$ teste de Johansen foi utilizado para analisar as relações econômicas de longo prazo entre as séries e, em caso de confirmação da cointegração, para verificar também quantas relações de longo prazo existem entre elas. $O$ teste foi realizado considerando três variáveis e os resultados são apresentados na Tabela 2.

Assim, observando-se os resultados apresentados na Tabela 2, conclui-se que há um único vetor de cointegração entre as variáveis analisadas, uma vez que a hipótese nula $h=0$ é rejeitada em favor da hipótese alternativa $h>0$; para um nível de significância de $1 \%$. E $\quad$ a hipótese nula $h \leq 1$, não foi rejeitada. Desta maneira, ao estimar o modelo de Auto Regressões Vetoriais, deve-se considerar a existência desta relação de longo prazo. Infere-se, então, que o modelo a ser estimado deve ser o VEC, para que sejam considerados tanto os aspectos de curto prazo quanto os de longo prazo.

Tabela 2. Resultados do teste de cointegração de Johansen para a cadeia da carne bovina.

\begin{tabular}{cccc}
\hline Hipótese Nula & Hipótese Alternativa & $\lambda_{\text {traco }}$ & Valor-p \\
\hline$h=0$ & $h>0$ & 51,260 & 0,005 \\
$h \leq 1$ & $h>1$ & 11,520 & 0,840 \\
$h \leq 2$ & $h>2$ & 2,955 & 0,871 \\
\hline
\end{tabular}

Fonte. Resultados da pesquisa

Uma última análise a ser realizada quanto à estabilidade do modelo estimado é feita através da observação dos coeficientes do VEC.

Tabela 3. Coeficientes dos modelos estimados

\begin{tabular}{lrrrr}
\hline Variável & Coeficiente & Erro padrão & Estatística-t & P-valor \\
\hline BOIVAR $\{1\}$ & 0,184 & 0,089 & 2,073 & 0,040 \\
BOIVAR $\{2\}$ & $-0,028$ & 0,086 & $-0,322$ & 0,748 \\
BOIATAC $\{1\}$ & 0,043 & 0,079 & 0,544 & 0,587 \\
BOIATAC $\{2\}$ & $-0,111$ & 0,071 & $-1,561$ & 0,120 \\
BOIPROD $\{1\}$ & 0,143 & 0,030 & 4,740 & 0,000 \\
BOIPROD $\{2\}$ & $-0,219$ & 0,046 & $-4,714$ & 0,000 \\
Constant & $-0,028$ & 0,086 & $-0,322$ & 0,748 \\
R1 & 0,014 & 0,086 & 0,166 & 0,868 \\
\hline
\end{tabular}

$\mathrm{O}$ VEC/VAR possui em sua estrutura problemas relacionados à multicolinearidade, sendo assim, o Teste $\mathrm{t}$ não é ideal para descartar parâmetros do modelo e não deve ser utilizado como medida de adequação de parâmetros, portanto, não foi feita nenhuma análise na tabela 3 neste sentido. Porém, é importante observar os valores dos coeficientes estimados, pois para garantir a estabilidade do modelo é necessário que os coeficientes estimados sejam menores que $|1|$ e observando a Tabela 3 , é possível observar que este critério foi atendido.

\subsection{Análise de Decomposição da Variância}

Para avaliar a importância de cada variável presente no sistema, foi analisada a decomposição da variância de cada uma das séries estimadas no VEC. Esta é uma medida da proporção de variação explicada pela própria série e pelas demais séries do 
modelo. Para fins de avaliação do poder de explicativo de uma série sobre a outra, foi considerado como proporção significativa, percentuais acima de $10 \%$.

A Tabela 4 mostra os resultados da Análise de Decomposição da Variância para o preço da carne de boi no varejo. Observa-se que o preço no atacado explica as variações do erro no preço no varejo, explicando em média $16,84 \%$ destas variações ao longo de doze meses. Cerca de $80 \%$ das variações são explicadas pela própria variável e menos de $3 \%$ explicadas por variações do preço ao produtor.

Tabela 4. Decomposição da Variância para o preço da carne bovina no varejo do período de 1997 a 2013

\begin{tabular}{ccccc}
\hline Meses & Desvio-padrão & PRODUTOR & ATACADO & VAREJO \\
\hline 1 & 0,024 & 0,000 & 8,323 & 91,677 \\
2 & 0,026 & 2,215 & 16,162 & 81,624 \\
3 & 0,026 & 2,409 & 16,050 & 81,541 \\
4 & 0,026 & 2,404 & 16,763 & 80,833 \\
5 & 0,026 & 2,434 & 16,798 & 80,768 \\
6 & 0,026 & 2,433 & 16,832 & 80,735 \\
7 & 0,026 & 2,434 & 16,843 & 80,723 \\
8 & 0,026 & 2,434 & 16,843 & 80,722 \\
9 & 0,026 & 2,434 & 16,845 & 80,721 \\
10 & 0,026 & 2,434 & 16,845 & 80,721 \\
11 & 0,026 & 2,434 & 16,845 & 80,721 \\
12 & 0,026 & 2,434 & 16,845 & 80,721 \\
\hline
\end{tabular}

Fonte. Resultado da Pesquisa

Os resultados da Tabela 5 correspondem à decomposição da variância para o preço no atacado, e mostram que os preços no varejo e ao produtor não têm efeito sobre o preço no atacado, ou seja, não ocorre transmissão de preços dos dois níveis de mercado para o atacado de forma significativa.

Tabela 5. Decomposição da Variância para o preço da carne bovina no atacado de 1997 a 2013

\begin{tabular}{ccccc}
\hline Meses & Desvio-padrão & PRODUTOR & ATACADO & VAREJO \\
\hline 1 & 0,036 & 0,000 & 100,000 & 0,000 \\
2 & 0,036 & 0,029 & 99,894 & 0,077 \\
3 & 0,036 & 0,079 & 99,715 & 0,206 \\
4 & 0,036 & 0,079 & 99,663 & 0,258 \\
5 & 0,036 & 0,086 & 99,654 & 0,260 \\
6 & 0,036 & 0,086 & 99,647 & 0,267 \\
7 & 0,036 & 0,087 & 99,646 & 0,267 \\
8 & 0,036 & 0,087 & 99,646 & 0,267 \\
9 & 0,036 & 0,087 & 99,646 & 0,268 \\
10 & 0,036 & 0,087 & 99,646 & 0,268 \\
11 & 0,036 & 0,087 & 99,646 & 0,268 \\
12 & 0,036 & 0,087 & 99,646 & 0,268 \\
\hline
\end{tabular}

Fonte. Resultado da Pesquisa 
Os resultados apresentados na Tabela 6 mostram que apenas o preço no atacado tem efeito sobre o preço ao produtor, explicando $65,71 \%$ das variações no preço ao produtor ao longo dos doze meses. $\mathrm{O}$ varejo explica menos de $1 \%$ destas variações, e a própria variável explica $33,8 \%$.

Tabela 6. Decomposição da Variância para o preço da carne bovina ao produtor de 1997 a 2013

\begin{tabular}{ccccc}
\hline Meses & Desvio Padrão & PRODUTOR & ATACADO & VAREJO \\
\hline 1 & 0,030 & 35,118 & 64,865 & 0,018 \\
2 & 0,031 & 34,052 & 65,875 & 0,073 \\
3 & 0,031 & 33,831 & 65,718 & 0,451 \\
4 & 0,031 & 33,828 & 65,720 & 0,452 \\
5 & 0,031 & 33,806 & 65,715 & 0,479 \\
6 & 0,031 & 33,806 & 65,713 & 0,481 \\
7 & 0,031 & 33,805 & 65,713 & 0,482 \\
8 & 0,031 & 33,805 & 65,713 & 0,482 \\
9 & 0,031 & 33,805 & 65,713 & 0,482 \\
10 & 0,031 & 33,805 & 65,713 & 0,482 \\
11 & 0,031 & 33,805 & 65,713 & 0,482 \\
12 & 0,031 & 33,805 & 65,713 & 0,482 \\
\hline
\end{tabular}

Fonte. Resultado da Pesquisa

Sendo assim, observa-se que o segmento mais exógeno é o atacado, pois suas variações de preço causam mudanças nos outros dois segmentos, porém é pouco impactado pelos outros segmentos.

\subsection{Análise da Função Impulso-Resposta}

Considerando que apenas as variações dos preços no atacado são transmitidas de forma significativa para os demais elos, conforme a análise de decomposição da variância, foram analisados apenas os resultados da função impulso-resposta dos preços ao atacado sobre os preços no varejo e ao produtor e ao próprio atacado. A Figura 1 permite visualizar os resultados dos choques nos preços do atacado sobre todos os três setores. As respostas ao impulso tiveram sinais esperados.

Figura 1. Função Impulso-resposta do preço ao atacado sobre todos os segmentos

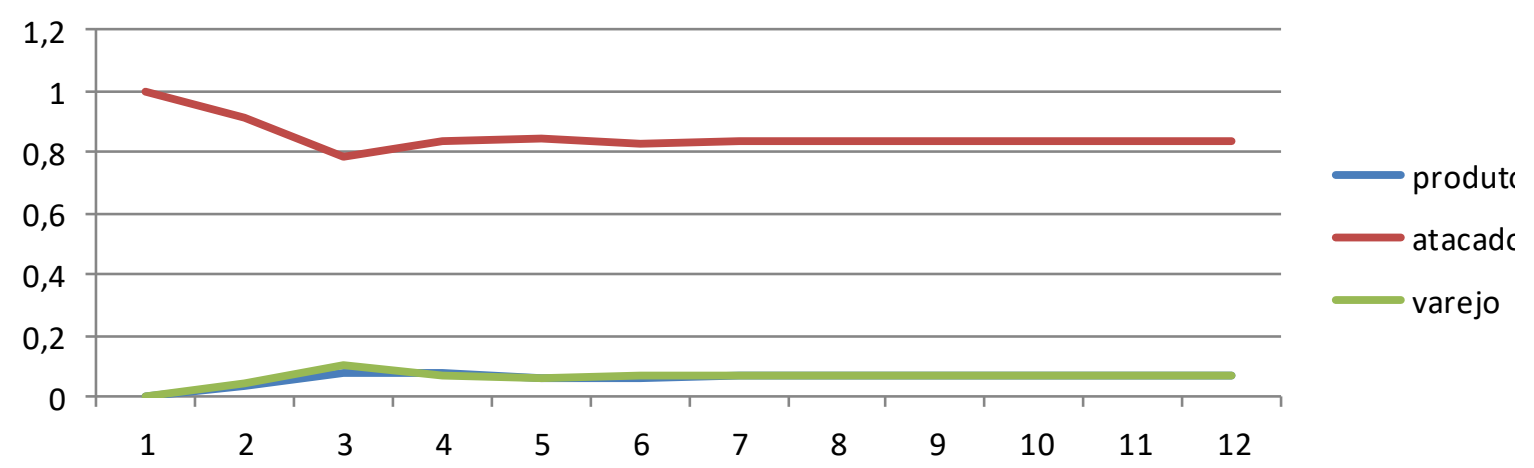

Fonte. Resultado da Pesquisa 
Um choque sobre seu próprio setor tende a ter efeito permanente de 0,83 , indicando que a reposta ao choque é de $83 \%$. Tanto para o varejo quanto para o produtor uns choques nos preços do atacado tendem a ter um efeito permanente bastante pequeno e similar de 0,06 , indicando que a resposta do varejo e do produtor a choques no atacado é de $6 \%$.

\section{CONCLUSÕES}

O presente estudo teve como principal objetivo analisar a elasticidade de transmissão de preços entre os elos de mercado, dentro da cadeia de carne bovina. Os resultados do trabalho permitiram confirmar a hipótese inicial de que o atacado é o grande responsável pela transmissão de preços para os níveis do varejo e do produtor. Verificou-se isso na cadeia bovina através dos cálculos apresentados. A hipótese inicial de que o atacado é o principal elo transmissor de preços se deve ao fato de o atacado contar com a presença de grandes frigoríficos, com elevado poder de barganha, além de ser o principal detentor de informações acerca dos demais elos. Além disso, outra explicação em relação ao atacado ser o maior responsável na transmissão de preços no período estudado, deve-se ao fato de o consumo de carne bovina ser consolidado no Brasil.

Ressalta-se que o presente trabalho envidou esforços no sentido de estudar o comportamento da cadeia bovina através da revisão bibliográfica e do levantamento de informações estatísticas, bem como aplicar técnicas de econometria de séries temporais para quantificar as elasticidades de transmissão de preços entre os elos de mercado. No entanto, fica como sugestão para trabalhos futuros uma investigação mais detalhada acerca do dimensionamento e do funcionamento dos sistemas agroindustriais das carnes bovina, para que se possam encontrar explicações complementares para o comportamento dos preços na comercialização agrícola do produto analisado.

\section{REFERÊNCIAS}

ABIEC - Associação Brasileira das Indústrias exportadoras de carne em 2014. Disponível em: http://www.abiec.com.br/Imprensa.aspx.pdf. Acesso 25/03/2019.

BACCHI, M. R. P., Previsão de preços de bovino, suíno e frango, com modelos de séries temporais. 1994. Tese (Doutorado em economia) Universidade de São Paulo, São Paulo, 1994.

BACCHI, M. R. P. Previsão de preços de bovino, suíno e frango, com modelos de séries temporais. 1994. Tese (Doutorado em economia) Universidade de São Paulo, São Paulo, 1994.

BARROS, G. S. A. C. Transmissão de preços pela Central de Abastecimento de São Paulo, Brasil. Revista Brasileira de Economia, v.44, n1, p.5-20, jan/mar.1990.

BARROS, G. S. A. C; ADAMI, A. C. O; ZANDONÁ, N. F. Embarques dos produtos do agronegócio recuam em 2014. CEPEA/ESALQ/USP. Piracicaba/SP, (2015). 
BARROS, G. S. A. C. Agronegócio Brasileiro, perspectivas, desafios e uma Agenda para seu desenvolvimento. CEPEA/LES-ESALQ/USP. Piracicaba/SP, (2006).

BARROS, G. S. A. C. Economia da Comercialização Agrícola, CEPEA/LESESALQ/USP, Piracicaba /SP, 221 p. (2007).

BUENO, R. L. S. Econometria de séries temporais. São Paulo: Thomson-Learning, 2011.

CEPEA - Centro de Estudos Avançados em Economia Aplicada. Disponível em: < Http://cepea.esalq.usp.br/pib/>.Acesso em 15/09/2015.

DICKEY, D. A.; FULLER, W.A. "Likelihood Ratio Statistics For Autoregressive Time Series With A Unit Root," Econometrical, 49, 1057-1072, 1981

EMBRAPA - Empresa Brasileira de Pesquisa Agropecuária. Pesquisas agropecuárias 2014. Disponível em: <https://www.infoteca.cnptia.embrapa.br/infoteca/bitstream/doc/658189/1/DOC442009. pdf $>$. Acesso em 15 de outubro de 2015.

ENDERS, W. Appied Econometric Times Series. New York: Jonh Wiley, 1995. 433 p.

GARDNER, B. L. The Farm-Retail price Spread in a Competitive Food Industry. American Journal of Agricultural Economics, v. 57, n. 3, p. 399-409, 1975.

GUJARATI, D. N. Econometria Básica. $4^{\mathrm{a}}$ ed. Rio de Janeiro: Elsevier Editora LTDA, 2006.

IBGE - Instituto Brasileiro de Geografia e Estatística. SIDRA: Pesquisa da Pecuária Municipal (PPM) - 2015. Disponível em: < http://www.agricultura.gov.br/portal/>. Acesso em: 12 de agosto de 2015.

JOHANSEN, S. Likelihood-Based Inference in Cointegrated Vector Autoregressive Models. Oxford: Clarendon Press. 1995.

MANKIW, N. G. Introdução à economia: princípios de micro e macroeconomia. Rio de Janeiro: Campus, 2010.

MARGARIDO, M. A. Transmissão de preços internacionais de suco de laranja para preços ao nível de produtor de laranja no Estado de São Paulo. Instituto de Economia Agrícola. Coleção Estudos Agrícolas 6. São Paulo-SP: Ática, 2000.

MENDES, J. T. G., PADILHA JR., J. B. Agronegócio: uma abordagem econômica. Pearson Prentice Hall, 2007. 369 p.

PEREIRA, B. D. Agropecuária de Mato Grosso: Velhas questões de uma nova economia. Cuiabá-MT: EdUFMT, 2012. 136 p. 
PINDYCK, R. S.; RUBINFELD, D. L. Microeconomia, $7^{\circ}$ ed. São Paulo: Pearson Education do Brasil, 2010.

PIZA, C. T.; WELSH, R. W. Introdução à Análise da Comercialização. 1968. In: BARROS, G. S. C. Economia da Comercialização Agrícola. Universidade de São Paulo - USP, Piracicaba, 1968.

SANTANA, A. C. de. Métodos Quantitativos em Economia: Elementos e Aplicações. Belém: UFRA, 2003. 484p.

ZYLBERSZTAJN, D. Economia das Organizações. In: ZYLBERSZTAJN, D; NEVES, M. F. (Orgs.) Economia e Gestão dos Negócios Agroalimentares. São Paulo: Pioneira, 2000. 